\title{
Macromolecular Crystallization and Crystal Perfection
}

\author{
NAOMI E. CHAYEN \\ Biomolecular Medicine, Department of Surgery and Cancer, \\ Imperial College London, UK \\ JOHN R. HELLIWELL \\ School of Chemistry, University of Manchester, UK \\ EDWARD H. SNELL
}

Hauptman-Woodward Medical Research Institute, Buffalo, USA 


\section{Contents}

Preface $\quad$ ix

Acknowledgements $\quad$ xi

\section{PART I INTRODUCTION AND OVERVIEW}

1. Introduction 3

1.1 Crystal growth 4

1.2 Diffraction techniques 6

1.2.1 X-rays 6

$\begin{array}{lll}1.2 .2 & \text { Neutrons } & 7\end{array}$

1.3 Crystal volume and quality 9

1.3.1 Short-range order (intermolecular) 10

1.3.2 Long-range order (domain structure) 14

1.3.3 The combination of short- and long-range order 16

$\begin{array}{lll}1.4 & \text { Chapter summary } & 17\end{array}$

\section{PART II CRYSTALLIZATION}

2. Crystallization theory 21

2.1 Chapter summary 23

3. Practical methods of crystallization 24

$\begin{array}{lll}3.1 \text { Batch } & 24\end{array}$

$\begin{array}{ll}3.2 & \text { Vapour diffusion } \\ 3.3 & 25\end{array}$

3.3 Dialysis 26

$\begin{array}{lll}3.4 & \text { Free interface diffusion } & 26\end{array}$

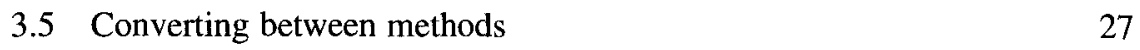

$\begin{array}{ll}3.6 & \text { Chapter summary } \\ \end{array}$

4. Screening 29

4.1 Screens: problems and new developments $\quad 29$

4.2 Automation and miniaturization of screening procedures 33

4.2.1 The effect of high-throughput: crystallization robotics $\quad 34$

4.2.2 Analysis of large quantities of crystallization data $\quad 34$

$\begin{array}{lll}4.2 .3 & \text { Experiment volume considerations } & 37\end{array}$

4.2.4 Imaging and monitoring of crystallization trials $\quad 37$

$\begin{array}{lll}4.3 & \text { Chapter summary } & 37\end{array}$ 
5. Optimization 38

5.1 Practical uses of the crystallization phase diagram 38

5.2 Methods for separating nucleation and growth $\quad 40$

$\begin{array}{lll}5.2 .1 & \text { Seeding } & 41\end{array}$

5.2.2 Dilution techniques $\quad 42$

5.3 The application of light-scattering techniques 44

5.3.1 Static light scattering $\quad 44$

5.3.2 Dynamic light scattering $\quad 45$

$\begin{array}{lll}5.4 & \text { Chapter summary } & 47\end{array}$

6. Strategies to apply when high-quality crystals $\begin{array}{ll}\text { cannot be obtained } & 48\end{array}$

$\begin{array}{ll}6.1 \text { Introduction } & 48\end{array}$

6.2 Non-covalent modification of the sample $\quad 48$

6.3 Covalent modification of the sample 49

6.3.1 Reductive methylation $\quad 49$

6.3.2 General chemical modification $\quad 50$

6.4 Mutagenesis, domain refinement and homologues $\quad 50$

$\begin{array}{lll}6.4 .1 & \text { Surface-entropy reduction } & 50\end{array}$

6.4.2 Proteolysis $\quad 51$

6.4.3 Orthologues and homologues $\quad 51$

6.5 Antibody fragments $\quad 52$

6.6 Chapter summary $\quad 52$

7. Membrane proteins 53

$\begin{array}{lll}7.1 & \text { Introduction } & 53\end{array}$

$\begin{array}{lll}7.2 & \text { Crystallization } & 53\end{array}$

7.2.1 Screening with detergents $\quad 55$

7.2.2 Lipidic cubic-phase crystallization $\quad 55$

7.2.3 Antibody fragment approaches $\quad 56$

7.3 Characterization using neutron-enhanced contrast 56

$\begin{array}{ll}7.4 \text { Chapter summary } & 56\end{array}$

8. Alternative approaches $\quad 58$

$\begin{array}{ll}8.1 \text { Gel growth } & 58\end{array}$

$\begin{array}{ll}8.2 \text { Microgravity } & 59\end{array}$

$\begin{array}{lll}8.2 .1 & \text { Interferometry } & 59\end{array}$

$\begin{array}{ll}\text { 8.2.2 Depletion zone } & 60\end{array}$

$\begin{array}{ll}8.3 \text { Microfluidics } & 61\end{array}$

8.4 Magnetic and electric fields $\quad 62$

$\begin{array}{ll}8.5 & \text { Chapter summary } \\ \end{array}$ 


\section{PART III DIFFRACTION}

9. Experimental aspects 67

9.1 The diffraction pattern 67

9.2 Structural detail $\quad 70$

9.3 Determining structure when the resolution is not ideal $\quad 72$

9.4 How accurate is the structure?

$\begin{array}{ll}9.5 & \text { Chapter summary }\end{array}$

10. Analysis of the molecular short-range order 78

10.1 Structural data 78

10.2 Thermal motion and diffuse scattering 79

10.2.1 Diffuse scattering as a source of measurement error in the Bragg intensities 81

10.3 Chapter summary 82

11. Analysis of long-range order 83

11.1 Reflection profiling $\quad 83$

11.2 Topography $\quad 88$

11.2.1 Photography-based topography $\quad 89$

11.2.2 Digital-based topography 90

11.3 Reciprocal-space mapping 91

11.4 Combinational analysis and chapter summary 94

12. Macromolecular crystals and twinning 96

$\begin{array}{lll}12.1 & \text { Historical examples } & 97\end{array}$

12.2 Types of twinning 98

12.3 The twin fraction and testing for twinned data 102

12.4 Using twinned data 104

12.5 Overcoming twinning in crystals 105

12.6 How prevalent is twinning? 109

$\begin{array}{ll}12.7 & \text { Chapter summary } \\ & 110\end{array}$

13. Other macromolecular crystal diffraction disorders 112

$\begin{array}{lll}13.1 & \text { Introduction } & 112\end{array}$

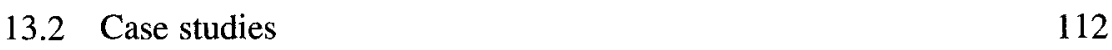

$\begin{array}{ll}13.3 \text { Chapter summary } & 118\end{array}$

14. Degradation and improvement of crystal perfection 119

$\begin{array}{ll}14.1 \text { Ageing } & 119\end{array}$

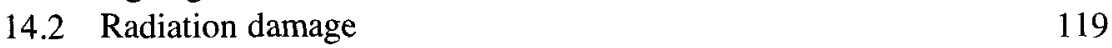

$\begin{array}{ll}14.3 \text { Cryo-cooling } & 124\end{array}$

$\begin{array}{ll}14.4 & \text { Dehydration and humidity control } \\ 14.5 & 127\end{array}$

14.5 Chapter summary 131 
15. Unusual diffraction geometries

16. Making the most of difficult crystals - beamline and detector optimization

16.1 Introduction

16.2 Geometry and end-station instrumentation

16.3 Signal and noise considerations

16.4 Very small crystal volume 'microcrystals'

16.5 Phasing and the available instrumentation

16.6 Robotics, telepresence and remote access

16.7 More specialized applications

16.8 Chapter summary

17. Protein powders - making the most of tiny crystallites in bulk 151

17.1 Introduction

17.2 Quantitative protein powder analyses have opened up in recent years

17.3 Structure determination of unknown proteins?

17.4 Characterization of protein crystal polymorphism 155

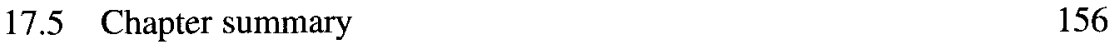

18. Complementary techniques 158

18.1 Chapter summary 159

\section{PART IV THE FU'TURE}

19. The X-ray laser and the single molecule - no crystal needed? 163

20. Overall summary and future thoughts 168

Glossary of abbreviations, terms and symbols $\quad 170$

Abbreviations 170

$\begin{array}{ll}\text { General } & 170\end{array}$

$\begin{array}{ll}\text { Synchrotron sources } & 171\end{array}$

$\begin{array}{ll}\text { Neutron sources } & 171\end{array}$

Terms 171

Crystallization $\quad 171$

$X$-ray analysis $\quad 172$

Symbols $\quad 173$

Crystallization and crystal-growth-monitoring symbols $\quad 173$

Diffraction symbols $\quad 174$

References $\quad 175$

$\begin{array}{ll}\text { Index } & 219\end{array}$ 